
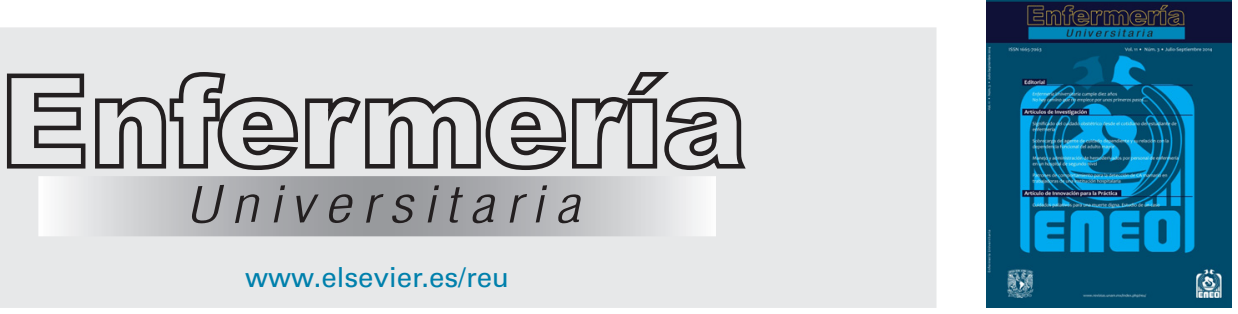

ARTÍCULO DE INVESTIGACIÓN

\title{
Adherencia al tratamiento en enfermedad cardiovascular: rediseño y validación de un instrumento
}

\section{B. Andrade Méndez ${ }^{\mathrm{a}, *}$ y V. Céspedes Cuevas ${ }^{\mathrm{b}}$}

a Programa de Maestría en Enfermería, Universidad Nacional de Colombia, Bogotá D. C., Colombia

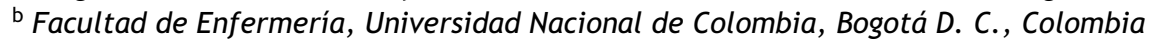

Recibido el 20 de enero de 2017; aceptado el 3 de octubre de 2017

Disponible en Internet el 4 de noviembre de 2017

\author{
PALABRAS CLAVE \\ Enfermedades \\ cardiovasculares; \\ Adherencia al \\ tratamiento; \\ Validez de las \\ pruebas; \\ Confiabilidad; \\ Colombia
}

\begin{abstract}
Resumen
Objetivo: Determinar la validez de constructo y confiabilidad del «Cuestionario para medir la adherencia al tratamiento de los pacientes con enfermedad cardiovascular».

Métodos: Estudio de tipo psicométrico. La muestra seleccionada fue de 250 pacientes con diagnóstico de enfermedad cardiovascular asistentes a una institución hospitalaria de tercer nivel de atención en la ciudad de Neiva (Colombia) en el primer semestre de 2016. Se desarrollaron cronológicamente diferentes fases investigativas: análisis del concepto, exploración y confirmación del constructo.

Resultados: A través del análisis del concepto se estableció como teoría de soporte la «autoeficacia» con sus tres componentes teóricos, seis dimensiones temáticas transversales y los atributos esenciales de la adherencia al tratamiento, los cuales se evidenciaron en la reformulación de los 38 ítems de la versión final del instrumento. La muestra en su mayoría estuvo conformada por personas mayores de 60 años, factor de riesgo predominante de hipertensión arterial y diagnóstico médico más frecuente de enfermedad coronaria. El análisis factorial exploratorio reportó seis factores con una varianza total explicada de $61.83 \%$. La matriz multirasgo-multimétodo demostró altas correlaciones intradimensión, lo cual evidenció la validez convergente del instrumento. El alfa de Cronbach obtenido fue de 0.92 para la escala total y dimensional por encima de 0.80 .
\end{abstract}

\footnotetext{
* Autor para correspondencia.

Correo electrónico: bandradem@unal.edu.co (B. Andrade Méndez).

La revisión por pares es responsabilidad de la Universidad Nacional Autónoma de México.
} 


\section{KEYWORDS}

Cardiovascular disease; Treatment adherence; Tests validity; Reliability; Colombia

\section{PALAVRAS-CHAVE}

Doenças cardiovasculares; Aderência ao tratamento; Validação das provas; Confiabilidade; Colômbia
Conclusiones: La versión actual del instrumento es válida y homogénea para la medición de la adherencia al tratamiento de los pacientes con enfermedad cardiovascular; útil para la investigación y la práctica clínica.

(c) 2017 Universidad Nacional Autónoma de México, Escuela Nacional de Enfermería y Obstetricia. Este es un artículo Open Access bajo la licencia CC BY-NC-ND (http://creativecommons.org/licenses/by-nc-nd/4.0/).

Adherence to treatment in cardiovascular disease: Re-design and validation of an instrument

\begin{abstract}
Objective: To determine the construct validity and reliability of the "Questionnaire to assess the adherence to treatment in patients with cardiovascular disease'.

Methods: This is a psychometric study. The selected sample included 250 patients diagnosed with cardiovascular disease and treated in a third-level-attention hospital institution in Neiva, Colombia during the first semester of 2016. The diverse research stages were chronologically developed as concept analysis, exploration, and construct confirmation.

Results: Through the concept analysis process, "self-efficacy" was established as a supporting theory with three theoretical components, six transversal thematic dimensions, and the essential attributes to the adherence to treatment, which were evidenced in the reformulation of the 38 items of the final version of the instrument. The sample was mainly constituted by persons older than 60 of age, which is a risk factor for hypertension and coronary problems. The exploratory factor analysis yielded 6 factors explaining $61.83 \%$ of the total variance. The multi-method and multi-treatment matrix demonstrated high intra-dimension correlations, and thus, the convergent validity of the instrument was evidenced. Cronbach alpha was 0.92 for the total scale, and 0.80 for the scale dimensions.

Conclusions: The present version of the instrument is valid and homogenous for the assessment of adherence to treatment in patients suffering from cardiovascular disease, and it is useful for the clinical practice and research.

(c) 2017 Universidad Nacional Autónoma de México, Escuela Nacional de Enfermería $\mathrm{y}$ Obstetricia. This is an open access article under the CC BY-NC-ND license (http://creativecommons.org/licenses/by-nc-nd/4.0/).
\end{abstract}

Aderência ao tratamento em doença cardiovascular: Redesenho e validação de um instrumento

\section{Resumo}

Objetivo: Determinar a validação de constructo e confiabilidade do «Questionário para medir a aderência ao tratamento dos pacientes com doença cardiovascular».

Métodos: Estudo de tipo psicométrico. A amostra selecionada foi de 250 pacientes com diagnóstico de doença cardiovascular, pacientes de uma instituição hospitalar de terceiro nível de atenção na cidade de Neiva (Colômbia) no primeiro semestre de 2016. Desenvolveram-se cronologicamente diferentes fases pesquisadoras: análise do conceito, exploração e confirmação do constructo.

Resultados: Através da análise do conceito estabeleceu-se como teoria de suporte a «autoeficácia» com seus três componentes teóricos, seis dimensões temáticas transversais e os atributos essenciais da aderência ao tratamento, os quais se evidenciaram na reformulação dos 38 itens da versão final do instrumento. A amostra em sua maioria esteve conformada por pessoas idosas de 60 anos, fator de risco predominante de hipertensão arterial e diagnóstico médico más frequente de doença coronária. A análise fatorial exploratória registou seis fatores com uma variância total explicada de 61.83\%. A matriz multirasgo-multimétodo demonstrou altas correlações intradimensão, o qual evidenciou a validação convergente do instrumento. A alfa de Cronbach obtida foi de 0.92 para a escala total e dimensional por cima de 0.80 .

Conclusões: A versão atual do instrumento é válida e homogénea para a medição da aderência ao tratamento dos pacientes com doença cardiovascular; útil para a pesquisa e a prática clínica. (c) 2017 Universidad Nacional Autónoma de México, Escuela Nacional de Enfermería y Obstetricia. Este é um artigo de acesso aberto distribuído sob a licença de Creative Commons CC BY-NC-ND (http://creativecommons.org/licenses/by-nc-nd/4.0/). 


\section{Introducción}

Actualmente, las enfermedades cardiovasculares son reconocidas como un problema importante de salud pública a nivel mundial, con incremento de las tasas de morbimortalidad y la prevalencia de factores de riesgo. La OMS reporta en el 2012 una mortalidad total de $\mathbf{1 7 . 5}$ millones de personas por esta causa, representa el $31 \%$ de la mortalidad mundial ${ }^{1}$. A nivel macroeconómico, los expertos prevén que en los próximos 20 años como consecuencias de estas patologías la producción mundial presentará pérdidas por más de US\$ 46 billones, resaltan que en la Región de las Américas las muertes son prematuras, es decir, se producen en personas de 30 a 69 años $^{2}$. Colombia como país en vías de desarrollo no es ajeno a esta realidad, ya que entre el 2005 y 2013 la enfermedad cardiocirculatoria fue la principal causa de muerte en la población general, generó 146.16 muertes por cada 100000 habitantes y el $16.13 \%$ de todos los años de vida potencialmente perdidos ${ }^{3}$, con lo que se demuestra el impacto de la carga global de enfermedad en esta población. Es evidente que los pacientes con ECV saturan los servicios de salud, para los cuales no se cuenta con programas de prevención y control específicos; sumado a lo anterior, la alta prevalencia demanda recursos humanos y financieros que ponen en una situación de alerta a los sistemas de salud ${ }^{4}$.

Las personas que presentan enfermedades crónicas requieren de tratamientos de larga duración para su adecuada evolución y control; los cuales deben ser mantenidos de manera sistemática durante toda la vida y exigen un comportamiento responsable y persistente para el logro de los objetivos en salud ${ }^{5}$. Es necesario enfatizar que la adherencia al tratamiento de las personas con enfermedades crónicas en los países desarrollados se estima en un $50 \%$ y en los países en vías de desarrollo las tasas son aún menores ${ }^{6}$. Es claro que para muchos pacientes es difícil seguir las recomendaciones y la deficiente adherencia a los tratamientos a largo plazo, compromete gravemente la efectividad de estos y del fármaco ${ }^{7}$, de manera que es un tema fundamental para la salud de la población desde la perspectiva de la calidad de vida y de la economía de la salud ${ }^{8}$.

La adherencia al tratamiento es definida por la $\mathrm{OMS}^{6}$ como el grado en que el comportamiento de una persona - tomar el medicamento, seguir un régimen alimentario y ejecutar cambios del modo de vida- corresponde con las recomendaciones acordadas de un prestador de asistencia sanitaria. La anterior definición está enmarcada dentro del concepto de cumplimiento, el cual implica la obediencia y la expectativa de que los pacientes pasivamente sigan las órdenes otorgadas por el prestador de salud. Al respecto Cohen ${ }^{9}$ define la adherencia como persistencia en la práctica y el mantenimiento de las conductas de salud deseados, como producto de la participación activa y el acuerdo; con énfasis en el desarrollo de una relación concordante y la toma de decisiones compartidas entre el paciente y el profesional de la salud. A su vez, Orueta et al. ${ }^{10}$, hacen referencia al papel preponderante que ejerce la motivación en el proceso de la adherencia y la fuerte influencia de factores como las creencias, los valores, la sintomatología percibida, la relación médico-paciente, el apoyo familiar-social, entre otros.

El fenómeno de la adherencia al tratamiento a nivel empírico presenta diversidad de métodos de medición formulados por diferentes disciplinas relacionadas con la salud de las personas, los cuales miden directa o indirectamente el fenómeno, detectan las ventajas y limitantes al utilizar cada uno de ellos o el uso combinado de estos métodos. En este sentido se hace mención a los métodos electrónicos (MEMS), instrumentos de autorreporte, informes de farmacia y pruebas específicas de laboratorio; el primero de ellos es muy costoso, poco accesible y la información es limitada con respecto a identificar si verdaderamente el paciente ingiere el medicamento y la dosis correcta ${ }^{7}$. Con relación a los instrumentos de autoinforme los definen como costo-efectivos y costo-eficientes, para tener acceso a la información y asumir si el paciente realmente es adherente al tratamiento ${ }^{11,12}$. Finalmente, se identifica que los resultados clínicos son medidas indirectas de la adherencia, mientras que los comportamientos del paciente como la pérdida de peso, el ejercicio y el tomar la medicación, son medidas directas de una verdadera adherencia $^{13}$.

Dentro de los instrumentos de autoinforme más referenciados y validados por diferentes estudios a nivel mundial, se encuentra el test de Morisky Grenn ${ }^{14,15}$, el cual presenta problemas de validez al sobreestimar la no adherencia y evaluar exclusivamente la adherencia al tratamiento farmacológico ${ }^{16}$. A nivel local, se han desarrollado investigaciones tendentes a la validación de instrumentos que evalúan la adherencia al tratamiento, como el instrumento desarrollado por Bonilla y Gutiérrez ${ }^{17}$ que mide los factores relacionados con la adherencia, el instrumento para la adherencia de pacientes con falla cardiaca ${ }^{18}$, el instrumento Martín Bayarré Grau en pacientes hipertensos ${ }^{19}$ y el uso del resultado de enfermería «conducta terapéutica: enfermedad o lesión», que corresponde a la Clasificación de Resultado de Enfermería o Nursing Outcomes Classification en pacientes hipertensos de la ciudad de Bucaramanga $\left(\right.$ Colombia) ${ }^{20}$.

Ante la necesidad de tener una medición válida del fenómeno de la adherencia al tratamiento, Achury ${ }^{21}$ diseñó el «Cuestionario para medir la adherencia al tratamiento de pacientes con enfermedad cardiovascular», el cual consta de 29 ítems que valoran la adherencia al tratamiento farmacológico y no farmacológico. Presenta validez facial y de contenido llevado a cabo con seis expertos y en 380 personas, el índice de kappa de Fleiss general mostró un acuerdo sustancial tanto en la comprensión como en la claridad del cuestionario, reportan una comprensibilidad superior al $95 \%$. Con relación a la validez de constructo, el análisis factorial con rotación Varimax arrojó una estructura de seis componentes que dan cuenta del $57.36 \%$ de la varianza explicada. Sin embargo, se identificó la necesidad de analizar la naturaleza conceptual que revelaría aproximadamente la mitad del constructo no explicado. A su vez, la confiabilidad general del instrumento presenta un alfa de Cronbach de 0.837 ; aunque se reportan dos dimensiones con confiabilidad débil y una con confiabilidad pobre.

Con base en las fortalezas del cuestionario desarrollado por Achury ${ }^{21}$ y de reconocer la necesidad de ahondar principalmente en el análisis conceptual de los factores identificados, se decide llevar a cabo el presente estudio que tiene como objetivo determinar la validez de constructo y confiabilidad del «cuestionario para medir la 
adherencia al tratamiento de los pacientes con enfermedad cardiovascular».

\section{Métodos}

El presente estudio es de tipo psicométrico, el cual aborda el desarrollo, la validación y la evaluación de instrumentos ${ }^{22}$. La investigación se llevó a cabo en el primer semestre del año 2016, se tomó como población a todas las personas (hombres y mujeres) que presentaban enfermedad cardiovascular, que acudieron a consulta al servicio de cardiología en el Hospital Universitario de Neiva (Colombia), institución de tercer nivel de atención. En cuanto al cálculo de la muestra se tomó como referente el modelo de determinación de tamaño de muestra en población finita propuesto por Tristán ${ }^{23}$; en este modelo el tamaño de la muestra se obtuvo en función de la fracción de desviación estándar que se acepte como error de aproximación para el análisis, por lo que se estableció como criterio teórico aceptable $(0.5)$ en términos de la desviación estándar. Finalmente, la muestra correspondió a un total de 250 pacientes, con un nivel de confianza de $95 \%$. Sumado a lo anterior, se consideró para efecto de obtener resultados representativos para el análisis factorial exploratorio la recomendación de abordar muestras superiores a 200 participantes $^{24}$. El tipo de muestreo fue por conveniencia.

Los criterios de inclusión fueron: pacientes (hombres y mujeres) mayores de 18 años, tener diagnóstico confirmado de enfermedad cardiovascular e indicación de tratamiento farmacológico y no farmacológico por más de seis meses. Asimismo, conservación de la esfera mental que les permitiera autodiligenciar el instrumento. Por su parte, se excluyeron los pacientes con diagnóstico de falla cardiaca quienes tienen recomendaciones particulares y exclusivas del manejo de la enfermedad, evaluadas a través de instrumentos específicos para esta patología.

El instrumento que se tomó como base para la validación de constructo fue el «Cuestionario para medir la adherencia al tratamiento de pacientes con enfermedad cardiovascular», consta de 29 ítems agrupados en 6 dimensiones ${ }^{21}$. Los primeros 27 ítems presentan una escala Likert con seis opciones de respuesta y los dos restantes son de selección múltiple. Los resultados oscilan entre 29 y 174 puntos, un mayor puntaje es el reflejo de una mejor adherencia al tratamiento. Es de anotar que con el desarrollo de la presente investigación se pretendió llevar a cabo las modificaciones necesarias a las pruebas planteadas para mejorar sus propiedades psicométricas.

En la presente investigación se desarrollaron secuencialmente las fases de análisis del concepto desde la teoría de soporte, exploración y verificación del constructo y finalmente, análisis de la confiabilidad a través del coeficiente de alfa de Cronbach.

Para el análisis de resultados se construyó una base de datos en el programa Microsoft office Excel 2013 y se procesaron a través del programa estadístico SPSS versión 15.0.

El presente estudio es considerado sin riesgo según la declaración de Helsinki, ya que no se realizó ninguna intervención sobre las personas. A su vez, se contó con el aval del comité ético de la Universidad Nacional de Colombia y del Hospital Universitario de Neiva. Previo al diligencia- miento del instrumento se procedió a explicar los alcances de la investigación, firma del consentimiento informado por parte de los pacientes, con respeto a los principios éticos de justicia, autonomía, beneficencia y no maleficencia.

\section{Resultados}

A continuación se presentan los principales resultados como producto de la ejecución sistemática de las fases planteadas con anterioridad.

\section{Análisis del concepto desde la teoría de soporte}

Esta fue la fase inicial de la presente investigación, en la cual se llevó a cabo la actualización del concepto de adherencia terapéutica enfocado a la salud cardiovascular, se tomó como referente la metodología de análisis del concepto de Walker y Avant $^{25}$. Se realizó una revisión exhaustiva en las bases de datos electrónicas EBSCO, Pubmed/Medline, CINHAL, se tomó como límites de búsqueda artículos publicados desde el 2004 hasta el 2015, que presentaran criterios de coherencia interna y externa; se seleccionaron 19 artículos que permitieron clarificar los atributos específicos de la adherencia al tratamiento como productos de resultados sólidos de investigación. De igual modo, se retomaron los análisis de concepto realizados por Shay ${ }^{26}$, Cohen ${ }^{9}$, Barbosa et al. ${ }^{27}$, Bayhakki ${ }^{28}$, como insumo para la selección de los atributos del concepto.

La adherencia al tratamiento es un concepto amplio, evidencia la relación directa entre procesos cognitivos y la participación activa por parte de las personas, de forma que le permite discernir, tomar decisiones y realizar acciones de autocuidado para el mantenimiento y mejora de su salud ${ }^{29}$. También, la adherencia está relacionada directamente con la autoeficacia de las personas ${ }^{30}$, entendida esta como los juicios que hace la persona de sus capacidades para organizar y ejecutar sus acciones. A su vez, se conceptualiza como la relación recíproca entre la persona, el ambiente y el comportamiento; se demuestra que a través del pensamiento reflexivo, el uso del conocimiento, las destrezas y otras herramientas de autoinfluencia la persona decide cómo comportarse.

Dentro de los atributos críticos o esenciales que definen el concepto fundamentado por la evidencia empírica se encuentra la motivación ${ }^{31}$, la autoconfianza ${ }^{32}$, las creencias $^{33}$, los conocimientos ${ }^{34}$, la participación activa, la permanencia y la persistencia en el tratamiento.

El paso siguiente fue contrastar los ítems originales del instrumento base con los atributos detectados en la revisión; se determinó que el instrumento incorporaba parcialmente conceptos de la teoría de autoeficacia (expectativas de autoeficacia), faltaba incluir la dimensión de expectativas de resultado y percepción del riesgo o susceptibilidad percibida; por lo tanto es relevante tener en cuenta desde el desarrollo teórico y empírico como lo demuestra la revisión bibliográfica. A su vez, se plantearon modificaciones en la redacción en los ítems a fin de evidenciar como atributo central la participación activa y la capacidad percibida del paciente cardiovascular. Por otra parte, la inclusión de nuevos ítems se hizo necesaria para evidenciar explícita 
e implícitamente los atributos esenciales del concepto de adherencia.

Como producto de esta fase se genera una nueva versión del instrumento con 38 ítems, con opción de respuesta tipo Likert (nunca, casi nunca, pocas veces y siempre), dirigido a pacientes con enfermedad cardiovascular que evalúa conjuntamente los aspectos del tratamiento farmacológico y no farmacológico. La propuesta generada presenta los tres componentes de la teoría de autoeficacia (expectativas de autoeficacia, expectativas de resultado y susceptibilidad percibida), así como las seis dimensiones temáticas transversales relacionadas con el uso y seguimiento farmacológico, capacidad de realizar ejercicio físico, dieta, manejo del estrés, necesidad de apoyo y control de factores de riesgo.

Posteriormente, en el trabajo de campo, se llevó a cabo la revisión de la historia clínica de los pacientes, esta aportó información relacionada con los datos demográficos y variables contextuales que influencian la adherencia al tratamiento. Al respecto de los 250 pacientes que conformaron la muestra, el $51 \%$ pertenece al sexo femenino, $71 \%$ son mayores de 60 años, con una edad mínima de 21 años y una máxima de 96 años. Los factores de riesgo observados para enfermedad cardiovascular demostraron que el $90 \%$ de los pacientes presentaron hipertensión arterial, 18\% diabetes mellitus tipo ॥, 10\% dislipidemia, 9\% enfermedad renal crónica, $8 \%$ obesidad y $6.4 \%$ de los pacientes padecen hipotiroidismo.

El diagnóstico médico más frecuente entre los participantes de la investigación fue la enfermedad coronaria con $68.8 \%$, clasificada dentro de esta a los pacientes con angina, infarto agudo de miocardio, cardiopatía isquémica y postoperatorio de revascularización miocárdica. Con una frecuencia de $12.4 \%$ se presentan los pacientes con diagnóstico de arritmias cardiacas, $8.4 \%$ con crisis hipertensivas y el restante $10.4 \%$ distribuidos en miocardiopatías, enfermedad valvular y vasculopatías.

\section{Exploración del constructo}

En esta fase se llevó a cabo el análisis factorial exploratorio con extracción por componentes principales y rotación Varimax ${ }^{35}$. Como actividad previa al análisis factorial se aplicaron pruebas de linealidad para identificar la viabilidad de análisis posteriores ${ }^{36}$. Dentro de ellas se menciona el test KMO (Kaiser, Meyer y Olkin), el cual relaciona los coeficientes de correlación observados entre las variables y los coeficientes de correlación parcial entre las variables. Cuanto más cerca de uno tenga el valor obtenido del test KMO, más alta es la relación entre las variables. EL KMO calculado para esta prueba corresponde a 0.875 el cual es notable y un nivel de significación de 0.000 , indicativo de significación estadística para llevar a cabo el análisis factorial.

Se tomó como criterio de extracción de factores valores propios superiores a uno (tabla 1). Se aprecia el listado de los autovalores de la matriz de varianzas-covarianzas y del porcentaje de varianza que representa cada uno de ellos. Los autovalores expresan la cantidad de la varianza total que está explicada por cada factor y los porcentajes de varianza explicada asociados a cada factor se obtienen a través de la división del autovalor por la suma de autovalores. El análisis factorial arrojó una estructura de seis componentes que dan cuenta del $61.83 \%$ de la varianza explicada la cual es satisfactoria y suficiente.

Para el análisis de la matriz de componentes rotados, se tomaron como significativas las cargas factoriales superiores a 0.3 en valor absoluto (tabla 2 ), los cuales se describen a continuación:

El primer factor agrupó los ítems del 19 al 26, correspondientes en su totalidad al componente teórico de expectativas de resultado, en el cual están inmersas las dimensiones temáticas de uso y seguimiento farmacológico, capacidad de realizar ejercicio físico, dieta saludable y manejo del estrés. Este factor se denominó Expectativas de resultado en el tratamiento farmacológico y no farmacológico, incluye ítems que miden la percepción del paciente con relación a los resultados positivos cuando este ejecuta comportamientos ligados a la adherencia al tratamiento.

El segundo factor agrupa los ítems que hacen parte de la dimensión temática de uso y seguimiento farmacológico (ítems 1, 8, 9, 10, 27, 30 y 31). Por consiguiente se denominó Expectativas de autoeficacia en el tratamiento farmacológico y seguimiento que incluye ítems estrechamente relacionados con el consumo de medicamentos.

El tercer factor agrupa ítems que hacen parte de la dimensión temática de capacidad de realizar ejercicio físico y necesidad de apoyo (ítems 5, 11, 12, 13, 29 y 32). Este factor se denominó expectativas de autoeficacia con el ejercicio físico y necesidad de apoyo, integra ítems que evalúan la percepción del paciente en el reconocimiento de las habilidades para realizar ejercicios y los beneficios otorgados por la ejecución de este.

El cuarto factor agrupa ítems que hacen parte de la dimensión temática de dieta saludable y control de factores de riesgo (ítems 2, 3, 4, 15, 16, 17,18 y 28). Este factor se denominó expectativas de autoeficacia con la alimentación y permanencia en el tratamiento, vincula ítems que evalúan la capacidad para llevar a cabo recomendaciones sobre la nutrición adecuada para el paciente cardiovascular, propias para el control de la patología.

El quinto factor agrupa ítems que hacen parte de la dimensión temática de uso y seguimiento farmacológico, capacidad de realizar ejercicio físico, dieta saludable y control de factores de riesgo (ítems 33, 34, 35, 36, 37, 38). Este factor se denomina percepción del riesgo, integra ítems que presentan una correlación fuerte relacionados con la capacidad del paciente para reconocer los riesgos que asume si no se adhiere a las recomendaciones acordadas con el personal de salud y las posibles complicaciones de hábitos inadecuados.

Por último, el sexto factor agrupa ítems que hacen parte de la dimensión temática de manejo del estrés $(6,7$ y 14). Este factor se denomina expectativas de autoeficacia para el manejo del estrés, vincula ítems que evalúan la capacidad del paciente para manejar las situaciones estresantes.

\section{Análisis confirmatorio}

En primer lugar se realizó el análisis de validez discriminante el cual permite distinguir variables que agrupan ítems y que difieren de otros agrupamientos. Esta técnica se basa en el análisis con una matriz de correlaciones para 
Tabla 1 Varianza total explicada

\begin{tabular}{|c|c|c|c|c|c|c|c|c|c|}
\hline \multirow[t]{2}{*}{$\begin{array}{l}\text { Compo } \\
\text { nente }\end{array}$} & \multicolumn{3}{|c|}{ Autovalores iniciales } & \multicolumn{3}{|c|}{$\begin{array}{l}\text { Sumas de extracción de } \\
\text { cargas al cuadrado }\end{array}$} & \multicolumn{3}{|c|}{$\begin{array}{l}\text { Sumas de rotación de cargas } \\
\text { al cuadrado }\end{array}$} \\
\hline & Total & $\%$ de varianza & $\%$ acumulado & Total & $\%$ de varianza & $\%$ acumulado & Total & $\%$ de varianza & $\%$ acumulado \\
\hline 1 & 10.899 & 28.682 & 28.682 & 10.899 & 28.682 & 28.682 & 5.108 & 13.442 & 13.442 \\
\hline 2 & 3.788 & 9.968 & 38.651 & 3.788 & 9.968 & 38.651 & 4.378 & 11.522 & 24.964 \\
\hline 3 & 2.863 & 7.535 & 46.186 & 2.863 & 7.535 & 46.186 & 4.247 & 11.175 & 36.139 \\
\hline 4 & 2.199 & 5.786 & 51.971 & 2.199 & 5.786 & 51.971 & 4.221 & 11.108 & 47.247 \\
\hline 5 & 2.030 & 5.342 & 57.314 & 2.030 & 5.342 & 57.314 & 3.164 & 8.325 & 55.573 \\
\hline 6 & 1.719 & 4.524 & 61.838 & 1.719 & 4.524 & 61.838 & 2.381 & 6.265 & 61.838 \\
\hline 7 & 1.342 & 3.532 & 65.370 & & & & & & \\
\hline 8 & 1.226 & 3.227 & 68.597 & & & & & & \\
\hline 9 & 1.094 & 2.879 & 71.475 & & & & & & \\
\hline 10 & 1.043 & 2.744 & 74.219 & & & & & & \\
\hline 11 & 0.923 & 2.428 & 76.646 & & & & & & \\
\hline 12 & 0.700 & 1.842 & 78.488 & & & & & & \\
\hline 13 & 0.595 & 1.566 & 80.054 & & & & & & \\
\hline 14 & 0.558 & 1.469 & 81.523 & & & & & & \\
\hline 15 & 0.504 & 1.326 & 82.850 & & & & & & \\
\hline 16 & 0.498 & 1.310 & 84.159 & & & & & & \\
\hline 17 & 0.480 & 1.263 & 85.422 & & & & & & \\
\hline 18 & 0.448 & 1.180 & 86.602 & & & & & & \\
\hline 19 & 0.435 & 1.146 & 87.748 & & & & & & \\
\hline 20 & 0.417 & 1.098 & 88.846 & & & & & & \\
\hline 21 & 0.367 & 0.965 & 89.812 & & & & & & \\
\hline 22 & 0.362 & 0.952 & 90.764 & & & & & & \\
\hline 23 & 0.345 & 0.908 & 91.672 & & & & & & \\
\hline 24 & 0.322 & 0.847 & 92.519 & & & & & & \\
\hline 25 & 0.303 & 0.799 & 93.318 & & & & & & \\
\hline 26 & 0.294 & 0.774 & 94.092 & & & & & & \\
\hline 27 & 0.280 & 0.737 & 94.829 & & & & & & \\
\hline 28 & 0.267 & 0.702 & 95.531 & & & & & & \\
\hline 29 & 0.235 & 0.620 & 96.150 & & & & & & \\
\hline 30 & 0.232 & 0.610 & 96.760 & & & & & & \\
\hline 31 & 0.208 & 0.548 & 97.308 & & & & & & \\
\hline 32 & 0.197 & 0.519 & 97.827 & & & & & & \\
\hline 33 & 0.172 & 0.452 & 98.279 & & & & & & \\
\hline 34 & 0.152 & 0.400 & 98.678 & & & & & & \\
\hline 35 & 0.139 & 0.366 & 99.044 & & & & & & \\
\hline 36 & 0.130 & 0.343 & 99.387 & & & & & & \\
\hline 37 & 0.128 & 0.337 & 99.724 & & & & & & \\
\hline 38 & 0.105 & 0.276 & 100.000 & & & & & & \\
\hline
\end{tabular}

realizar diversos agrupamientos, de manera que optimice cada grupo en cuanto a sus correlaciones y los diferencie de los otros grupos. En la presente investigación se decidió hacer la identificación de agrupaciones de los ítems a partir del análisis ítem-total ${ }^{37}$ (correlación punto-biserial), así como tomar en cuenta que cada uno de los conjuntos de ítems agrupados se considera como una posible variable, si esta se compone de más de tres ítems y si la correlación positiva con el conjunto es superior a 0.196. Como se observa en la tabla 3, los seis factores cumplen con los criterios antes descritos, por lo que determinan que los ítems que lo componen se comportan como una dimensión.

Como estrategia de corroboración de la presencia de validez de constructo y discriminante, se llevó a cabo la construcción de la matriz multirrasgo-multimétodo bajo las directrices propuestas por Campbell y Fiske ${ }^{38}$; así como ubicar en la diagonal de la matriz los coeficientes de fiabilidad de cada factor y fuera de la diagonal las correlaciones entre los factores. Por consiguiente, se exigen altas correlaciones intradimensión como evidencia de la validez convergente y bajas correlaciones interdimensión cuando los rasgos son asumidos como diferentes, estos soportan la validez discriminante y confirman el constructo. Finalmente, se obtuvo una matriz que permitió observar las correlaciones altas de Pearson (superior a 0.196) dentro de cada componente teórico, lo que evidencia confirmación de la validez de constructo. De la misma manera, las correlaciones de Pearson bajas (inferiores a 0.196), demuestran que se trata de componentes teóricos (rasgos o atributos) diferentes, lo que da soporte a la confirmación de validez discriminante. 
Tabla 2 Matriz de componentes rotado

\begin{tabular}{|c|c|c|c|c|c|c|}
\hline Ítems & Factor 1 & Factor 2 & Factor 3 & Factor 4 & Factor 5 & Factor 6 \\
\hline $\begin{array}{l}\text { 20. Creo que las indicaciones del personal de salud en } \\
\text { relación con mi alimentación son importantes para el } \\
\text { control de mi enfermedad }\end{array}$ & 0.769 & & & & & \\
\hline
\end{tabular}

21. Pienso que el ejercicio físico indicado por el personal de salud es necesario para controlar mi enfermedad

25. Si consumo alimentos bajos en sal y grasa, disminuirá la probabilidad de alteraciones cardiovasculares

24. Si yo manejo apropiadamente las situaciones estresantes, controlare la tensión arterial

22. Si yo tomo los medicamentos indicados, voy a disminuir mis probabilidades de tener un ataque cardiaco

23. Si yo realizo ejercicio físico controlaré mi peso

19. Pienso que si tomo diariamente todos mis medicamentos de acuerdo con la orden médica me sentiré mejor

26. Si realizo ejercicio físico me sentiré más activo

8. Soy capaz de asistir a los controles programados por el personal de salud

30. Durante el tratamiento, dejo de tomar algunos medicamentos si me siento bien

31. Si en poco tiempo NO presento mejoría en mi salud, suspendo mis medicamentos

9. Soy capaz de realizarme los exámenes de laboratorio y otros exámenes en los periodos que los profesionales de salud me indican

27. Por alguna razón suspendo mis medicamentos sin la indicación del personal de salud

10. Estoy atento a cualquier signo o síntoma que demuestre un empeoramiento en mi estado de salud, como: dolor en el pecho, presión arterial alta o baja, palpitaciones, ahogo o fatiga, inflamación de brazos y piernas

1. Puedo tomar mis medicamentos en el horario establecido

13. Necesito que otras personas me recuerden que debo hacer el ejercicio físico ordenado por el personal de salud

32. Si en poco tiempo NO presento mejoría en mi salud, suspendo el ejercicio físico

29. Por alguna razón suspendo el ejercicio físico sin la indicación del personal de salud

5. A pesar de mi trabajo y oficios dedicaría tiempo para realizar ejercicio físico

11. Necesito que otras personas me recuerden que debo tomar mis medicamentos

12. Necesito que otras personas me recuerden que debo seguir la alimentación ordenada por el personal de salud

2. Evito en mi alimentación el consumo de grasa (pollo con piel, fritos, alimentos de paquete, leche entera, alimentos precocidos)

3. Puedo evitar en mi alimentación el consumo de harinas y azúcares

4. Soy capaz de consumir alimentos bajos en sal

28. Por alguna razón dejo de seguir las recomendaciones de la alimentación dadas por el personal de salud

0.764

0.760

0.745

0.733

0.724

0.479 
Tabla 2 (continuación)

\begin{tabular}{|c|c|c|c|c|c|c|}
\hline Ítems & Factor 1 & Factor 2 & Factor 3 & Factor 4 & Factor 5 & Factor 6 \\
\hline $\begin{array}{l}\text { 16. Cumplo con el tratamiento de mi enfermedad } \\
\text { aunque me parezca complicado }\end{array}$ & & & & 0.603 & & \\
\hline $\begin{array}{l}\text { 18. Soy capaz de seguir las indicaciones que me da el } \\
\text { personal de salud }\end{array}$ & & & & 0.595 & & \\
\hline $\begin{array}{l}\text { 15. Soy capaz de cambiar los comportamientos dañinos } \\
\text { para mi salud }\end{array}$ & & & & 0.586 & & \\
\hline $\begin{array}{l}\text { 17. Hago lo que está a mi alcance para mejorarme } \\
\text { cuando estoy enfermo }\end{array}$ & & & & 0.509 & & \\
\hline $\begin{array}{l}\text { 34. Es necesario suspender el uso del cigarrillo como } \\
\text { parte fundamental de mi tratamiento }\end{array}$ & & & & & 0.754 & \\
\hline $\begin{array}{l}\text { 35. Pienso que si NO consumo los medicamentos como } \\
\text { me han indicado, tengo probabilidad de presentar una } \\
\text { alteración en mi salud }\end{array}$ & & & & & 0.736 & \\
\hline $\begin{array}{l}\text { 33. Consumir bebidas alcohólicas aumentaría el riesgo } \\
\text { de presentar complicaciones en mi salud }\end{array}$ & & & & & 0.707 & \\
\hline $\begin{array}{l}\text { 36. Pienso que tengo riesgo de obesidad o de tener un } \\
\text { infarto en el corazón si consumo alimentos grasos, } \\
\text { salados y embutidos }\end{array}$ & & & & & 0.671 & \\
\hline $\begin{array}{l}\text { 37. Creo que tengo mayor probabilidad de tener } \\
\text { complicaciones cerebrales, renales con relación a } \\
\text { otras personas que NO tienen enfermedad } \\
\text { cardiovascular }\end{array}$ & & & & & 0.558 & \\
\hline $\begin{array}{l}\text { 38. Creo que NO realizar ejercicio físico me llevará a } \\
\text { tener mayor riesgo de complicaciones en mi salud }\end{array}$ & & & & & 0.536 & \\
\hline $\begin{array}{l}\text { 6. Puedo realizar actividades que me ayuden a manejar } \\
\text { el estrés o las situaciones tensionantes }\end{array}$ & & & & & & 0.841 \\
\hline $\begin{array}{l}\text { 7. Cuando estoy angustiado, hago algo que me ayude a } \\
\text { sentirme mejor }\end{array}$ & & & & & & 0.823 \\
\hline $\begin{array}{l}\text { 14. Es difícil manejar las situaciones tensionantes o que } \\
\text { me producen estrés }\end{array}$ & & & & & & 0.808 \\
\hline
\end{tabular}

Tabla 3 Correlaciones por cada uno de los factores

\begin{tabular}{lll}
\hline Factor & \# DE Ítems & $\begin{array}{c}\text { Ponderado de las } \\
\text { correlaciones }\end{array}$ \\
\hline Expectativas de resultado en el tratamiento farmacológico y no farmacológico & 8 & 0.688 \\
Expectativas de autoeficacia en el tratamiento farmacológico y seguimiento & 7 & 0.635 \\
Expectativas de autoeficacia con el ejercicio físico y necesidad de apoyo & 6 & 0.870 \\
Expectativas de autoeficacia con la alimentación y permanencia en el tratamiento & 8 & 0.658 \\
Percepción del riesgo & 6 & 0.573 \\
Expectativas de autoeficacia para el manejo del estrés & 3 & 0.697 \\
\hline
\end{tabular}

\section{Análisis de la confiabilidad}

La confiabilidad se orientó a medir la consistencia interna, la cual es óptima para la aplicación única de un test y se refiere al grado en que los ítems de una escala se correlacionan entre ellos. A su vez, si los puntos que componen una escala teóricamente miden el mismo constructo deben mostrar una alta correlación, es decir, la escala debe presentar un alto grado de homogeneidad ${ }^{39}$. La consistencia interna del instrumento se considerara aceptable cuando se encuentre entre 0.70 y 0.90 ; los valores de consistencia interna inferiores a 0.70 son indicativos de una pobre correlación entre los ítems.

El alfa de Cronbach general para el cuestionario de 38 ítems fue de 0.924 , el cual demuestra que los ítems de la escala se correlacionan entre ellos. Por consiguiente, los puntos que componen esta escala diseñada teóricamente miden el mismo constructo de la adherencia al tratamiento, presentan un grado óptimo de homogeneidad. Del mismo modo, permite apreciar que cada uno de los factores presenta una óptima fiabilidad, la cual es superior a 0.8 (tabla 4). 
Tabla 4 Confiabilidad de cada uno de los factores

\begin{tabular}{lll}
\hline Factor & N. $^{\circ}$ de ítems & Alfa de Cronbach \\
\hline 1 & 8 & 0.901 \\
2 & 7 & 0.860 \\
3 & 6 & 0.877 \\
4 & 8 & 0.881 \\
5 & 6 & 0.807 \\
6 & 3 & 0.834 \\
\hline
\end{tabular}

\section{Discusión}

Como parte de la valoración de la adherencia al tratamiento, se describen los autoinformes (autorreportes) como test de valoración que permiten identificar las conductas reportadas por los pacientes; estos tienen como ventajas la facilidad para su uso y reducción de los costos en su aplicación ${ }^{40}$. No obstante, se evidencia la necesidad de tener un patrón estándar que permita evaluar este fenómeno a través de una prueba que involucre el tratamiento farmacológico como el no farmacológico, y tome como pilar la participación activa del paciente ${ }^{41}$. Por lo anterior, el presente instrumento responde a las necesidades antes expuestas.

La actualización del concepto de adherencia terapéutica enfocado a la salud cardiovascular se llevó a cabo a través de la metodología de análisis del concepto de Walker y Avant ${ }^{25}$; permitió dilucidar que los ítems del instrumento base se encontraban redactados en términos de cumplimiento, lo cual se constituía en un reto, por lo tanto se considera que para tener un éxito en el tratamiento debe lograrse una participación activa de la persona ${ }^{9,42}$. Es de aclarar que el análisis de concepto es un proceso lingüístico riguroso, útil para determinar los atributos de un concepto que dependen del tiempo y contexto en que se define; genera la necesidad de que estos sean analizados periódicamente, para su continua revisión y perfeccionamiento ${ }^{43}$.

El instrumento original tomó como referente teórico la autoeficacia para la construcción de los ítems, se determinó que esta propuesta presentaba un solo componente de la teoría de autoeficacia (expectativas de autoeficacia), pendientes de incluir los componentes de expectativas de resultado y percepción del riesgo o susceptibilidad percibida, los cuales son importantes desde lo teórico y empírico como lo demuestra la revisión bibliográfica ${ }^{32,44}$. Por consiguiente, se reorganizó el instrumento en los tres componentes teóricos de autoeficacia y seis dimensiones temáticas. De igual forma, se realizaron modificaciones en la redacción de los ítems que demostraran como característica principal la participación activa, la capacidad percibida y el juicio de capacidad del paciente cardiovascular ${ }^{45}$.

La dimensión teórica de susceptibilidad percibida es entendida como la percepción subjetiva del riesgo de desarrollar una enfermedad y la aceptación de un diagnóstico. En este sentido, la persona que presenta negación ante un diagnóstico de enfermedad crónica, no experimenta síntomas graves y cree que se siente bien; tienen menor probabilidad de adherirse al tratamiento ${ }^{46}$. También, Mendoza et al., hacen referencia a la percepción de gravedad del paciente o el sentirse enfermo, como un catalizador que aumenta la probabilidad de cumplimiento de la terapia, haciéndole pensar que el tratamiento que le ha sido indicado será benéfico para su salud ${ }^{47}$.

Por otra parte, Ogedegbe et al. ${ }^{48}$ sustentan que la autoeficacia es un predictor conocido de una amplia gama de comportamientos en salud, el cual debe ser vinculado en estudios de adherencia al tratamiento. En este sentido, la percepción de autoeficacia desempeña un papel importante en la realización y permanencia de la ejecución de ejercicio físico $^{49}$, debido a que la autoeficacia actúa como intermediaria entre la intención de realizar la actividad física y el desarrollo posterior de la misma. Por su parte, Grembowski et al. ${ }^{50}$ evaluaron la relación entre la autoeficacia y el comportamiento en salud de la población adulta mayor en cinco hábitos: ejercicio, la ingesta de grasas en la dieta, control de peso, ingesta de alcohol y el hábito de fumar, determinado que las expectativas de autoeficacia y de resultado para estos comportamientos en salud son dependientes.

En la literatura se dispone de instrumentos diseñados bajo el modelo teórico de autoeficacia, los cuales determinan algunos elementos esenciales en la adherencia al tratamiento con medición independiente, algunos de ellos son: la escala de expectativas de resultados para el ejercicio $(\mathrm{OEE})^{51}$, la escala de autoeficacia de adherencia al medicamento (MASES) ${ }^{52}$, «Inventario de Autoeficacia Percibida para el Control del Peso» ${ }^{53}$, autoeficacia para la salud relacionada con la dieta y el ejercicio ${ }^{54}$, entre otros; los cuales hacen una medición parcializada del fenómeno de la adherencia y no evidencian los parámetros mínimos de estabilidad psicométrica necesarios que soporten su uso en procesos de investigación o en la práctica.

Al considerar el criterio de validez, la nueva versión del instrumento deja ver una estructura de seis factores que dan cuenta del $61.83 \%$ de la varianza explicada, presenta soporte teórico al interior de cada factor. A su vez, se hizo confirmación del constructo por medio de las pruebas de correlación punto-biserial y matriz multirasgo-multimetodo; que determinó validez convergente y discriminante. Cortada de Kohan ${ }^{55}$ hace mención de la necesidad de establecer una relación estrecha entre la teoría cognitiva y psicométrica, con el objeto de poner los test al servicio de la investigación e identificación de las aptitudes particulares de los sujetos evaluados.

Por último, la confiabilidad del instrumento base reporta un alfa de Cronbach general de 0.837 , pero con problemas en la consistencia interna demostrada en las dimensiones con valores de aceptable, débil y pobre, lo que indicó la necesidad de revisión y reformulación del cuestionario ${ }^{21}$; en cambio, la consistencia interna del instrumento actual obtuvo un coeficiente de alfa de Cronbach para los 38 ítems, de 0.924 para el instrumento total y un alfa superior a 0.8 en cada una de sus dimensiones; se demuestra así, que el nuevo instrumento presenta una alta correlación, es decir, la escala demuestra un alto grado de homogeneidad ${ }^{39}$.

\section{Conclusiones}

Las investigaciones de tipo psicométrico aportan a la disciplina de Enfermería herramientas sólidas, en el sentido de poner a disposición instrumentos construidos y validados sobre modelos teóricos en la determinación temprana de la adherencia al tratamiento farmacológico y no 
farmacológico de los pacientes con enfermedad cardiovascular. En este sentido, la teoría de la autoeficacia deja ver cómo las expectativas de autoeficacia, de resultado y la percepción del riesgo juegan un papel relevante en los comportamientos que adoptan las personas en relación con la salud; que favorecen la intervención en quienes presentan conductas relacionadas con una baja adherencia y que mantienen intervenciones de soporte en los pacientes adherentes. Es así como la identificación temprana de las creencias de las personas ayuda a superar los obstáculos y contribuye a la aceptación del tratamiento, que beneficie la calidad de vida del paciente y su familia.

Las propiedades psicométricas del «cuestionario para medir la adherencia al tratamiento de los pacientes con enfermedad cardiovascular», se destacan por ser una medida válida y confiable para aquellas personas que tienen indicado un tratamiento farmacológico y no farmacológico, que podrían estar en riesgo potencial de no adherirse. Por ende, se constituye en una herramienta relevante para conseguir medidas objetivas acerca de las expectativas del paciente sobre los riesgos asociados con la enfermedad cardiovascular, el beneficio del tratamiento para su calidad de vida y la prevención de complicaciones, y de sus aspectos motivacionales para superar los obstáculos comunes en el inicio de la terapia y mantener la conducta.

Finalmente, se pone a disposición de los profesionales de Enfermería un instrumento válido y confiable, que permite determinar la adherencia al tratamiento de los pacientes con patología cardiovascular en el ámbito clínico, así como en la asistencia primaria. Por tanto, provee de modelos para la identificación oportuna de necesidades de cuidado, brindándole elementos de peso para diseñar estrategias efectivas de intervención y mejorar la situación de salud de la comunidad que tiene a su cuidado.

\section{Responsabilidades éticas}

Protección de personas y animales. Los autores declaran que los procedimientos seguidos se conformaron a las normas éticas del comité de experimentación humana responsable y de acuerdo con la Asociación Médica Mundial y la Declaración de Helsinki.

Confidencialidad de los datos. Los autores declaran que han seguido los protocolos de su centro de trabajo sobre la publicación de datos de pacientes.

Derecho a la privacidad y consentimiento informado. Los autores han obtenido el consentimiento informado de los pacientes y/o sujetos referidos en el artículo. Este documento obra en poder del autor de correspondencia.

\section{Conflicto de intereses}

Los autores declaran no tener ningún conflicto de intereses.

\section{Agradecimientos}

A los pacientes que participaron de la presente investigación y funcionarios del Hospital Universitario de Neiva que aprobaron el desarrollo de esta investigación.

\section{Referencias}

1. Organización Mundial de la Salud. Enfermedades cardiovasculares, Nota descriptiva. Ginebra: OMS. 2015.

2. Organización Panamericana de la Salud. Plan de acción para la prevención y el control de las enfermedades no transmisibles en las Américas 2013-2019. Washington, DC: OPS, 2014.

3. Ministerio de Salud y Protección Social. Análisis de Situación de Salud. Colombia, 2015. Bogotá D.C., Colombia: Ministerio de salud y Protección Social; 2015.

4. Gómez H, Castro MV, Franco F, et al. La carga de la enfermedad en países de América Latina. Salud Publica Mex. 2011;2:72-7.

5. Martín Alfonso L, de los A, Grau Ábalo JA, et al. Marco conceptual para la evaluación y mejora de la adherencia a los tratamientos médicos en enfermedades crónicas. Rev Cubana Salud Pública. 2014;40:225-38.

6. Organización Mundial de la Salud. Adherencia a los tratamientos a largo plazo: pruebas para la acción. Washington, D.C.: OMS, 2004.

7. Nogues Solán X, Sorli Redó ML, Villar García J. Instrumentos de medida de adherencia al tratamiento. An Med Interna (Madrid). 2007;24:138-41.

8. Bowry AD, Shrank WH, Lee JL, et al. A systematic review of adherence to cardiovascular medications. J Gen Intern Med. 2011;26:1479-91, http://dx.doi.org/10.1007/ s11606-011-1825-3.

9. Cohen SM. Concept analysis of adherence in the context of cardiovascular risk reduction. Nurs Forum. 2009;44:25-36, http://dx.doi.org/10.1111/j.1744-6198.2009.00124.x.

10. Orueta R, Toledano P, Gómez R. Cumplimiento terapéutico. Actualización en medicina de familia. SEMERGEN. 2008;34:235-43, http://dx.doi.org/10.1016/ S1138-3593(08)71889-6.

11. Gonzalez JS, Schneider HE, Wexler DJ, et al. Validity of medication adherence self-reports in adults with type 2 diabetes. Diabetes Care. 2013;36:831-7, http://dx.doi.org/10.2337/dc12-0410.

12. Rolley JX, Davidson PM, Dennison CR, et al. Medication adherence self-report instruments implications for practice and research. J Cardiovasc Nurs. 2008;23:497-505, http://dx.doi.org/10.1097/01.JCN. 0000338931.96834.16.

13. Burke LE, Yang K, Acharya SD. Adherence to cardiovascular treatment regimens. In: Woods $S$, et al., editors. Cardiac Nursing. Philadelphia: Wolters Kluwer Health/Lippincott Williams \& Wilkins; 2010. pp. 885-901.

14. Yang A, Wang B, Zhu G, et al. Validation of chinese version of the morisky medication adherence scale in patients with epilepsy. Seizure. 2014;23:295-9, http://dx.doi.org/10.1016/j.seizure.2014.01.003.

15. Morisky DE, Ang A, Krousel-Wood M, et al. Predictive validity of a medication adherence measure in an outpatient setting. $J$ Clin Hypertens (Greenwich). 2008;10:348-54

16. Ávila CW, Aliti GB, Feijó MKF, et al. Adhesión farmacológica al anticoagulante oral y factores que influyen en la estabilidad del índice de estandarización internacional. Rev. Latino-Am. Enfermagem. 2011;19, 8 pantallas.

17. Bonilla C, Gutiérrez de Reales E. Desarrollo y características psicométricas del instrumento para evaluar los factores que influyen en la adherencia a tratamientos farmacológicos y no farmacológicos en personas con factores de riesgo de enfermedad cardiovascular. Av.Enferm. 2014;32:53-62, http://dx.doi.org/10.15446/av.enferm.v32n1.46035.

18. Achury-Saldaña DM, Sepúlveda-Carrillo GJ, RodríguezColmenares $\mathrm{S}$, et al. Validez y confiabilidad de un instrumento evaluativo de adherencia en pacientes con falla cardiaca. Enferm Glob. 2012;11:1-9, http://dx.doi.org/10.4321/S1695-61412012000200001. 
19. William-Martínez J, Villa-Perea J, Jaramillo J, et al. Validación del cuestionario de adherencia al tratamiento anti hipertensivo Martín Bayarré Grau. Rev Méd Risaralda. 2011;17:101-5.

20. Páez-Esteban A, Caballero-Díaz L, López-Barbosa N, et al. Validez de constructo de un instrumento de enfermería para evaluar adherencia al tratamiento antihipertensivo. Enferm Glob. 2014;13:37-47, http://dx.doi.org/10.6018/ eglobal.13.2.170111.

21. Achury Beltrán L. La entrevista motivacional y la adherencia de los pacientes en pos infarto agudo de miocardio. [tesis de Maestría]. Bogotá: Universidad Nacional de Colombia, Facultad de Enfermería; 2015.

22. Polit D, Hungler P, Palacios R, et al. Investigación científica en ciencias de la salud. Sexta Edición México: McGraw Hill Interamericana; 2000. p. 171-211.

23. Tristán LA. Cálculo del tamaño de muestra y establecimiento de criterios y el problema del tamaño de la población. Guía de usuario Kalt Criterial Versión 2. San Luis Potosí: Instituto de Evaluación e Ingeniería Avanzada S.C; 2003.

24. Kääriäinen $M$, Kanste $O$, Elo $S$, et al. Testing and verifying nursing theory by confirmatory factor analysis. J AdvNurs. 2011;67:1163-72, http://dx.doi.org/10.1111/ j.1365-2648.2010.05561.x.

25. Walker L, Avant KC. Strategies for theory construction in nursing. 5. th edition Upper Saddle River, NJ: Pearson Prentice Hall; 2011. p. 157-75.

26. Shay LE. A concept analysis: Adherence and weight loss. Nurs Forum. 2008;43:42-52, http://dx.doi.org/10.1111/ j.1744-6198.2008.00095.x.

27. Barbosa CD, Balp MM, Kulich K, et al. A literature review to explore the link between treatment satisfaction and adherence, compliance, and persistence. Patient Prefer Adherence. 2012:39-48, http://dx.doi.org/10.2147/PPA.S24752.

28. Bayhakki. Adherence:A concept analysis. Songklanagarind J Nurs. 2014:149-58.

29. Velandia A, Rivera L. Agencia de autocuidado y adherencia al tratamiento en personas con factores de riesgo cardiovascular. Rev salud pública. 2009;11:538-48.

30. Smith MJ, Liehr P. Middle range theory for nursing. Second Edition New York: Springer Publishing Company; 2008. p. 49-64.

31. Rodríguez-Bueno L, García-Barragán L. El modelo transteórico y adherencia terapéutica en adolescentes con diabetes mellitus tipo I. Rev Iberoam Psicol Salud: Ciencia y Tecnología. 2014;7:49-58.

32. Polsook R, Aungsuroch Y, Thanasilp S, et al. Validity and Reliability of Thai Version of Questionnaire Measuring Selfefficacy for Appropriate Medication Use Scale among Thai with post-myocardial infarction. Songklanakarin J. Sci. Technol. 2014;36:411-7.

33. Noar SM, Zimmerman RS. Health behavior theory and cumulative knowledge regarding health behaviors: Are we moving in the right direction? Health Educ Res. 2005;20:275-90, http://dx.doi.org/10.1093/her/cyg113.

34. Zhao S, Zhao H, Wang L, et al. Education is critical for medication adherence in patients with coronary heart disease. Acta Cardiol. 2015;70:197-204.

35. Carvajal A, Centeno C, Watson R, et al. ¿Cómo validar un instrumento de medida de la salud? An. Sist. Sanit. Navar. 2011;34:63-72.

36. Fuente-Fernández S. Análisis conglomerados. Madrid: UAM, Facultad de Ciencias Económicas y Empresariales; 2011.

37. Tristán LA. Diseño de pruebas (Parte 4). México: Instituto de Evaluación e Ingeniería Avanzada; 1999-2007, 13-17.

38. Campbell D, Fiske D. Convergent and discriminant validation by the multitrait-multimethod matrix. Psychol Bull. 1959;56:81-105.
39. Campo A, Oviedo H. Propiedades psicométricas de una escala: la consistencia interna. Rev. Salud pública. 2008;10: 831-9.

40. Rodríguez Chamorro MA, García-Jiménez E, Amariles $P$, et al. Revisión de test de medición del cumplimiento terapéutico utilizado en la práctica clínica. Aten Primaria. 2008;40:413-7, http://dx.doi.org/10.1157/13125407.

41. López-Romero L, Romero-Guevara S, Parra DI, et al. Adherencia al tratamiento: Concepto y medición. Hacia promoc. salud. 2016;21:117-37, http://dx.doi.org/10.17151/hpsal.2016.21.1.10.

42. Johnson $M$, Javalkar $K$, Van Tilburg $M$, et al. The relationship of transition readiness, self-efficacy, and adherence to preferred health learning method by youths with chronic conditions. J Pediatr Nurs. 2015;30:83-90, http://dx.doi.org/10.1016/j.pedn.2015.05.014.

43. Melo Fernandes M, Lima da Nóbrega MM, Ribeiro Garcia $\mathrm{T}$, et al. Análise conceitual: considerações metodológicas. Rev Bras Enferm. 2011;64:1150-6, http://dx.doi.org/10.1590/ S0034-71672011000600024.

44. Strecher V, deVellis BM, Becker MH, et al. The role of self-efficacy in achieving health behavior change. Healt Educ Q. 1986;13:73-92, http://dx.doi.org/10.1177/109019818601300108.

45. Olaz F, Silva M, Pérez E. Guía para la construcción de escalas de autoeficacia. Argentina: Universidad Nacional de Córdoba; 2001.

46. Ávila Sansores GM, Gómez-Aguilar P, Yam-Sosa AV, et al. Un enfoque cualitativo a las barreras de adherencia terapéutica en enfermos crónicos de Guanajuato, México. Aquichan. 2013;13:373-86.

47. Mendoza S, Muñoz M, Merino J, et al. Factores determinantes de cumplimiento terapéutico en adultos mayores hipertensos. Rev Méd Chile. 2006;134:65-71.

48. Ogedegbe G, Mancuso CA, Allegrante JP, et al. Development and evaluation of a medication adherence self-efficacy scale in hypertensive African-American patients. J Clin Epidemiol. 2003;56:520-9.

49. Bray SR, Gyurcsik NC, Culos-Reed SN, et al. An exploratory investigation of the relationship between proxy efficacy, self-efficacy and exercise attendance. J Health Psychol. 2001;6:425-34, http://dx.doi.org/10.1177/ 135910530100600405.

50. Grembowski D, Patrick D, Diehr P, et al. Self-efficacy and health behavior among older adults. J Health Soc Behav. 1993;34:89-104.

51. Resnick B, Zimmerman S, Orwig D, et al. Outcome expectations for exercise scale: utility and psychometrics. J Gerontol B Psychol Sci Soc Sci. 2000;55:S352-6.

52. Fernandez S, Chaplin W, Schoenthaler AM, et al. Revision and validation of the Medication Adherence Self- Efficacy Scale (MASES) in Hypertensive African Americans. J Behav Med. 2008;31:453-62, http://dx.doi.org/10.1007/s10865-008-9170-7.

53. Román Y, Díaz B, Cárdenas $M$. Construcción y validación del Inventario Autoeficacia Percibida para el Control de Peso. Clin Salud. 2007; 18:45-56.

54. Sallis Jf, Pinski Rb, Grossman Rm, et al. The development of the self-efficacy scales for health related diet and exercise behaviors. Health Educ Res. 1988;3:283-92, http://dx.doi.org/10.1093/her/3.3.283.

55. Cortada de Kohan N. Posibilidad de integración de las teorías cognitivas y la psicometría moderna. Interdisciplinaria. 2005;22:29-58. 